\title{
Funktioniert die europäische Lohnkoordinierung? Befunde aus der deutschen Metall- und Elektroindustrie
}

Um innerhalb der Europäischen Union (EU) Unterbietungswettbewerb zu verhindern, länderspezifische Inflationsunterschiede zu kompensieren und eine gleichmäßige Tarifentwicklung zu gewährleisten, bemühen sich die Gewerkschaften programmatisch um eine internationale Lohnkoordinierung. Doch während die europäische Integration die Verhinderung von Standortwettbewerb eher erschwert, zeigen die Initiativen für eine transnationale Lohnkoordinierung bisher einen nur begrenzten Erfolg. Anhand einer qualitativen Analyse der Lohnkoordinierung in der deutschen Metall- und Elektroindustrie zeigt der Beitrag, warum sich Gewerkschaften hierfür weiterhin engagieren, auch wenn sie noch weit davon entfernt sind, ihre gesetzten Ziele zu erreichen.

MARTIN SEELIGER

\section{Einleitung}

Mit der europäischen Wirtschafts- und Währungsunion hat sich der Stellenwert einer international koordinierten Lohnpolitik für die Gewährleistung nationaler Wettbewerbsfähigkeit nochmals signifikant erhöht. Das Ziel einer EU-weit koordinierten Lohnpolitik ist es, Differenzen beim Produktivitätszuwachs und mit Blick auf die Inflationsraten auszugleichen, um Unterbietungswettbewerb zu verhindern und makroökonomische Stabilität zu gewährleisten. Mit ihren Initiativen zur internationalen Lohnkoordinierung versuchen die Gewerkschaften seit Mitte der 1990er Jahre, diese Herausforderung aufzugreifen. Doch angesichts der institutionellen Heterogenität nationaler Lohnfindungssysteme und mangelnder tarifpolitischer Durchsetzungsfähigkeit waren diese Initiativen bislang wenig erfolgreich. Gleichzeitig halten die deutschen Gewerkschaften an der Notwendigkeit einer europäischen Koordinierung der Tarifpolitik fest und sehen sie als einen zentralen Bestandteil ihres europapolitischen Kerngeschäftes.

Dieses Spannungsverhältnis untersucht der folgende Artikel auf Basis einer qualitativen Fallstudie der Lohnkoordinierung in der deutschen Metall- und Elektroindustrie. Das anhaltende Engagement der Gewerkschaften in der Lohnkoordinierung erklärt sich - so ein zentraler Befund - aus einer akteurszentriert-handlungstheoretischen Perspektive (Abschnitt 4). Vorab erfolgt ein Problemaufriss so- wie ein kurzer Überblick über den Forschungsstand (2). Abschnitt 3 gibt Einblick in die empirische Analyse der zugrunde liegenden Untersuchung.

2. Problemaufriss und Forschungsstand

\subsection{Lohnkoordination als Sollbruchstelle fester Wechselkursregime}

Lohnfindung und Tarifpolitik sind das Kerngeschäft gewerkschaftlichen Engagements. Aus Sicht der Gewerkschaften überlappen sich hier zwei unterschiedliche Referenzsysteme "in einem Spannungsverhältnis zwischen den unmittelbaren Verteilungsinteressen der tarifpolitischen Akteure einerseits und den ökonomischen Steuerungsinteressen einer Gesellschaft andererseits" (Bispinck/Schulten 2006, S. 254).

Mit der europäischen Währungsunion hat sich die Bedeutung der Lohnpolitik für die Gewährleistung makroökonomischer Stabilität in der Eurozone signifikant erhöht. Während früher nationale Inflationsdynamiken über Aufund Abwertungen seitens der Zentralbanken korrigiert werden konnten, beeinflusst nunmehr die Lohnstückkosteninflation auch die Preisinflation. Will man die makroöko- 
nomische Stabilität als volkswirtschaftliche Zielgröße bejbehalten, sind die Tarifpartner somit gezwungen, die nationalen Lohnstückkosten an der Zielinflationsrate des gemeinsamen Währungsraums zu orientieren (Hancké 2013). Auf diese Weise wird die Lohnpolitik unter Bedingungen der Währungsunion (d. h. des Verlusts nationaler Abwertungsmöglichkeiten zugunsten der geldpolitischen Kompetenz der Europäischen Zentralbank (EZB) und des Fiskalpaktes) zu einem gewichtigen makroökonomischen Steuerungsinstrument.

Uber die Frage, wie wichtig die Fähigkeit zur effektiven Lohnkoordinierung für die Gewährleistung makroökonomischer Stabilität gemeinsamer Währungsräume tatsächlich ist, herrscht in der einschlägigen politökonomischen Literatur keineswegs Einigkeit. Schulten (2014) beispielsweise sieht die Bedeutung der Lohnstückkosten für die makroökonomischen Ungleichgewichte als vielfach überschätzt. Als standortspezifischer Wettbewerbsfaktor sei nicht nur der Preis der Arbeit einschlägig, sondern auch nicht-preislichen Aspekte wie die Kontinuität der Produktion oder die Produktqualität spielten hier eine Rolle. Anders als Hancké (2013) hinterfragt Schulten auch den unmittelbaren Kausalzusammenhang von Lohn- und Preisentwicklung und betont zudem die Bedeutung der Lohnentwicklung für die Einkommensverteilung. Vor diesem Hintergrund gelangt er zu dem Schluss, dass die Lohnpolitik als alleiniges Instrument zum Ausgleich makroökonomischer Ungleichgewichte überfordert sei (vgl. auch Müller et al. 2016). Doch unabhängig von den genannten - und zweifelsfrei vorhandenen - komplementären Einflussfaktoren geht dieser Beitrag von einer zentralen Bedeutung der Lohnstückkostenentwicklung - und folglich der Notwendigkeit ihrer Koordinierung - aus.

Die ökonomische Wirklichkeit sieht derweil anders aus: Während die nominalen Lohnstückkosten von 2000 bis zur Krise von 2008 in Frankreich immerhin um 20\%, in Italien um $25 \%$ und in Spanien sogar um über $30 \%$ anstiegen, stagnierten sie in der Bundesrepublik im gleichen Zeitraum. Wie Höpner (2014, S. 317) bemerkt, hätten die nominalen Lohnstückkosten in der Eurozone im Zeitraum von 1999 bis 2008 um $22 \%$ wachsen müssen, um die Zielinflationsrate der EZB in Höhe von $2 \%$ einzuhalten. Diese Entwicklungen identifiziert Hancké (2013, S. 321) als Kernproblem der Währungsunion: “Since its inception in 1999, European Monetary Union has witnessed an increased divergence of inflation and wages, as well as of economic performance more generally in the single currency area."

Der Entwicklung in Deutschland kommt hierbei aufgrund seiner hohen Wirtschaftsleistung, die sich zu einem wesentlichen Teil im Export in die anderen Länder der Eurozone abbildet, eine Schlüsselbedeutung zu. Diese starke Außenhandelsorientierung ist seit Jahrzehnten (genauer: seit der Nachkriegsära) ein charakteristisches Merkmal des deutschen Wirtschaftsmodells (Horn 2010). Nachdem der Anteil der Exporte an der Gesamtwirtschaft vor allem nach der Wiedervereinigung abgenommen hatte, stieg er im Laufe der 2000er Jahre rapide an und belief sich ab 2011 auf über $50 \%$ des Bruttoinlandprodukts (BIP) (Bispinck/ Schulten 2014, S. 14). Gegenüber der Jahrtausendwende hat sich zudem der Exportüberschuss auf ca. $217 \mathrm{Mrd}$ € zum Ende des hier betrachteten Untersuchungszeitraums im Jahr 2014 vervierfacht (Der Spiegel 13/2015, S. 23). Dies ist knapp skizziert - der ökonomische Hintergrund, vor dem die folgende Darstellung die praktische Umsetzung der Lohnkoordinierung untersucht. Dies geschieht am Beispiel der europäischen Metall- und Elektroindustrie. Mit dem Flächentarifvertrag der deutschen Metall- und Elektroindustrie richtet sich die Aufmerksamkeit auf dasjenige Segment der deutschen Volkswirtschaft, in dem die größten Exporte in den Euroraum verzeichnet werden.

\subsection{Geschichte der Lohnkoordinierung im europäischen Metallsektor}

Initiativen grenzüberschreitender Lohnkoordinierung werden unter europäischen Gewerkschaften schon seit einigen Jahrzehnten diskutiert (Pen 1964). Vor dem Hintergrund der Währungsunion gewannen Initiativen zur Abstimmung nationaler Tarifabschlüsse neue politische Aufmerksamkeit. Hierbei kommt dem Europäischen Metallgewerkschaftsbund (EMB) eine tragende Rolle zu. Nach längeren Auseinandersetzungen einigten sich nationale Gewerkschaften auf Initiative ihres Dachverbandes auf die sog. Koordinierungsregel, der zufolge Lohnforderungen mindestens der Addition von Produktivitätszuwächsen und dem Ausgleich der Inflationsrate entsprechen müssen. Wie im Jahr 1996 auf einer Sitzung des EMB in Frankfurt a. M. beschlossen, sollten die beteiligten europäischen Gewerkschaften diese Forderungen in ihren lokalen Tarifverhandlungen durchsetzen, um dem im gemeinsamen Markt verstärkten Standortwettbewerb zu begegnen.

Zur Operationalisierung entsprechender Abstimmungsmaßnahmen wurde mit der Eucob@n-Datenbank ein System zum Monitoring von Tarifabschlüssen der beteiligten Länder etabliert (Schulten 2004, S. 292). Zum Zweck der Verstetigung ist weiterhin die Entsendung von Delegierten der europäischen Ebene in die nationalen oder regionalen Lohnverhandlungen vorgesehen (Gollbach 2005). Über den Modus der Ermittlung der Lohnforderungen bestehen im Koordinierungssystem der IndustriALL Europe als Nachfolgeorganisation des EMB jedoch Unklarheiten: sowohl hinsichtlich der zugrunde liegenden Produktivitätsrate (sektoral oder gesamtwirtschaftlich) als auch in Bezug auf die (entweder national variierende oder durch die EZB bei $2 \%$ fixierte) Inflationsrate.

1 Dass diese Rechnungsweise bis heute nicht von allen an den Koordinierungsinitiativen beteiligten Metallgewerkschaften geteilt wird, erschwert die Erreichung einheitlicher Ergebnisse von vornherein. 


\subsection{Stand der Forschung}

Als zentrales Feld gewerkschaftlicher Zusammenarbeit in der EU ist die europäische Lohnkoordinierung, die seit Einführung der Koordinierungsregel vor allem auf sektoraler Ebene Gestalt angenommen hat, seit Mitte der 1990er Jahre auch Thema sozialwissenschaftlicher Analysen (vgl. u. a. Johnston 2009; Traxler et al. 2008; Traxler/Brandl 2009). Eine transnationale Abstimmung der Lohnabschlüsse sei, so die Schlussfolgerung von Traxler et al. (2008, S. 106), am ehesten "auf der Grundlage nicht-hierarchischer, netzformiger Koordinierungsmechanismen" möglich. Empirische Analysen leisten hierbei meist eine indikatorengestützte Darstellung europäischer Lohnentwicklung und etwaiger Koordinierungserfolge (Glassner 2009). Nachdem in der Frühphase der Koordinierungsinitiativen seit Mitte der 1990er positive Einschätzungen überwogen, welche die Potenziale solcher Abstimmungen herausgestellt hatten (Schulten/Bispinck 1999; Marginson/ Traxler 2005), fallen die Bewertungen seit Mitte der 2000er Jahre - und vor allem nach der EU-weiten Wirtschaftskrise - vorsichtiger aus (Erne 2013; Sterkel/Wiedemuth 2011). Und tatsächlich haben sich Initiativen transnationaler Lohnkoordinierung in der EU - zumindest gemessen an den Zielgrößen der Verhinderung von Unterbietungswettbewerb sowie der Gewährleistung makroökonomischer Stabilität - bis heute als nicht besonders effektiv erwiesen. ${ }^{2}$ Neben den (noch fehlenden) unmittelbar tarifpolitischen Auswirkungen zählt aus Sicht der Gewerkschaften aber auch ein positiver „Prozessnutzen", welcher sich aus der Pflege internationaler Kontakte im Rahmen des europäischen Dachverbandes ergibt.

Die anvisierte Abstimmung von Löhnen im Einklang mit der Koordinierungsregel beinhaltet für die Gewerkschaften eine Reihe von Schwierigkeiten. Dies zeigt sich im Exportsektor anhand des „Anreizes", sich Wettbewerbsvorteile im Verhältnis $z u$ den ausländischen Konkurrenten zu sichern (Höpner 2013, S. 6). ${ }^{3}$ Eine zweite Problemstellung ergibt sich für die Koordinierungsinitiativen aus der Heterogenität nationaler Tarifsysteme in der Eurozone hinsichtlich der Mitgliederstärke von Arbeitgeber- und Gewerkschaftsverbänden, der jeweiligen Prozedur der Lohnfindung sowie ihrer Absicherung durch den Staat. Als dritte erschwerende Bedingung treten schließlich Sanktionen der Troika gegen die Euro-Krisenländer hinzu (Schulten/Müller 2013, S. 295). Angesichts solcher gravierender politökonomischen Differenzen innerhalb des Euroraumes ist eine synchrone Lohnund Tarifpolitik als unwahrscheinlich anzusehen.

Aus Sicht einer Reihe von Beiträgen macht sie dies jedoch nicht obsolet. Denn infolge der New Economic Governance, so Müller et al. (2016), gibt es weitere Gründe für die Notwendigkeit gewerkschaftlicher Lohnkoordinierung im Euroraum. Vor dem Hintergrunde staatlich verordneter Austerität werde die gewerkschaftliche Abstimmung von l.ohnforderungen zu einem zentralen Element für ein nachfrageorientiertes und lohngetriebenes Wachstumsmodell (vgl. Pernicka 2015). D. h. neben der Verhinderung von Unterbietungswettbewerb sowie der Gewährleistung ma- kroökonomischer Stabilität tritt unter diesen Bedingungen ein breiteres Kriterium für die Beeinflussung des lohnpolitischen Diskurses hinzu.

Das Augenmerk der Forschung hat sich bislang allerdings vorrangig auf die die konzeptionellen Fragen und Probleme der internationalen Lohnkoordinierung konzentriert. Schon weit seltener wurden ihre Auswirkungen empirisch untersucht. Gänzlich vermissen lässt die aktuelle Forschungslandschaft Untersuchungen, die sich für den qualitativen Prozess von Lohnkoordinierung im Rahmen konkreter Tarifverhandlungen interessieren. Die empirische Studie, auf der dieser Beitrag basiert, hat eine solche mikrodynamische Perspektive eingenommen und die Frage zu beantworten versucht, warum sich die europäischen Gewerkschaften für eine grenzüberschreitende Lohnkoordinierung engagieren, obwohl entsprechende Initiativen in der Vergangenheit nur bedingt erfolgreich waren und unter den gegebenen Bedingungen auch nur schwer zu verwirklichen sind.

\section{Lohnkoordinierung im deutschen Metallsektor - eine empirische Rekonstruktion}

Der vorliegende Text basiert auf einer Teilstudie gewerkschaftlicher Positionsbildung in der europäischen Tarifpolitik (vgl. Seeliger 2017). Der Untersuchungszeitraum reicht vom finalen Beschluss der Euroeinführung durch den Bundestag im Jahr 1998 bis 2013. Mit dem Ziel einer Rekonstruktion der spezifischen Sichtweisen der an den Initiativen beteiligten Akteure folgt die Darstellung einem akteurzentrierten, handlungstheoretischen Zugang (Joas/Beckert 2001). Die Auswahl der Interviewpartner erfolgte hierbei zum einen über professionelle Kontakte des Forschers; zum anderen auf die konkrete Empfehlung interviewter Personen. Die weiter unten zu Wort kommenden Gewerkschafterinnen und Gewerkschafter ${ }^{4}$ sind allesamt hauptamtlich im Bereich der Tarifpolitik beschäftigt. Ausgehend von der

2 Bei dieser kritischen Bilanz ist allerdings auch in Rechnung zu stellen, dass vor allem seit den 2000er Jahren mit der tarifpolitisch ausgehandelten Garantie etwa von Weiterbildungsansprüchen oder Betriebs- und Frühverrentungsmodellen qualitative Elemente derTarifpolitik an Bedeutung gewonnen haben, deren Durchsetzung nicht zuletzt auf Kosten der quantitativen Lohnentwicklung erfolgt ist

3 Diese Tendenz verstärkt im Untersuchungszeitraum (1998-2013, vgl. Abschnitt 3) eine wirtschaftliche Krise im Metallsektor, deren tarifpolitische Implikationen Bispinck und Dribbusch (2011) aufarbeiten.

4 Aus Gründen der Anonymität wird im Folgenden durchgängig die männliche Form verwendet. 
Analyse einschlägiger Veröffentlichungen wie internen und öffentlichkeitswirksamen Positionspapieren und Selbstdarstellungstexten in Print- und Onlineform war es das Ziel der 20 Experteninterviews mit Vertretern unterschiedlicher nationaler Gewerkschaftsorganisationen sowie Vertretern europäischer Dachverbände, die nominelle Programmatik mit der realen Lohnentwicklung zu kontrastieren und Einblicke zu gewinnen in die Herausforderungen tarifpolitischen Handelns unter Bedingungen der Wirtschafts- und Währungsunion. ${ }^{5}$

\subsection{Lohnkoordinierung in Zusammenarbeit von IG Metall und IndustriALL Europe}

Die politische Ökonomie der Bundesrepublik folgt nach $\operatorname{dem}$ Zweiten Weltkrieg dem Entwicklungspfad eines hochindustrialisierten Landes - hauptsächlich aufgrund ihrer Exportstärke. Die weltwirtschaftliche Rolle Deutschlands wurde intern durch eine Konstellation ermöglicht, die ihre sozialwissenschaftlichen Chronisten auch als Sozial- (oder später: Konflikt-) Partnerschaft beschrieben haben (Müller-Jentsch 2011). Ein stabiler arbeitspolitischer Bezugsrahmen ergab sich im Zusammenspiel starker Tarifpartner, die eine Beteiligung der Lohnabhängigen am gesellschaftlichen Wohlstand auf einem relativ hohen Niveau gewährleistete, und eine Beteiligung der Belegschaften durch die Mitbestimmung auf Betriebs- und Unternehmensebene.

Mit fast 2,3 Mio. Mitgliedern vertritt die IG Metall das größte Exportsegment der deutschen Wirtschaft. Ihre politische Orientierung folgt hierbei, so Rüb (2009, S. 144), einer "reformistisch-wirtschaftsdemokratisch ausgerichteten Agenda" mit dem Ziel einer "linkskeynesianistische[n] Reformalternative im Kapitalismus. "Bei den Gewerkschaften der Exportsektoren erkennen Experten ein genuines Interesse an der Erhaltung von Wettbewerbsfähigkeit (vgl. Thelen 2014, S. 36). Eine entsprechende Orientierung der IG Metall spiegelt sich traditionell in einem auf qualitative Wettbewerbsfähigkeit und Produktivitätssteigerung ausgerichteten Produktivismus (Rüb 2009, S. 145).

Seit den 1970er Jahren hat die IG Metall ihre europapolitischen Interessen zunächst im Europäischen Metallgewerkschaftsbund (EMB; vormals: Metallarbeiterbund) vertreten. Dieser fusionierte Ende 2012 gemeinsam mit der European Mine, Chemical and Energy Workers' Federation und der European Trade Union Federation for Textiles, Clothing and Leather zu dem umfassenderen Dachverband IndustriALL Europe (im Folgenden: IndustriALL). Ein Kernstück des europapolitischen Engagements stellt die in Abschnitt 2.2 durch den Dachverband IndustriALL beschriebene Initiative zur Lohnkoordinierung dar.

Hinsichtlich der grenzüberschreitenden Abstimmung von Lohnverhandlungen betont die IG Metall in einer Broschüre zum Thema, ,wie wichtig es ist, den Koordinierungsansatz nicht auf, Regeln' und ,Formeln zu beschränken, sondern auf einem politischen Ansatz zu bestehen" (IG Metall 2005, S. 21). Eine ähnlich klare Position bezieht auch der Europäische Metallgewerkschaftsbund, indem er sich von lediglich rhetorischen Bekenntnissen abgrenzt und den praktischen Charakter betont: "This wage coordination rule is a concrete political and policy tool towards employers, which will be actively used by the collective bargaining negotiators of all affiliated organisations" (EMB 2011, S. 12). Ein eindeutiges Bekenntnis zur Lohnformel (mindestens Produktivitätszuwachs plus Inflationsrate) findet sich auch in der Politischen Resolution 2011 - 2015 des neu gegründeten Verbandes IndustriALL Europe (2012a, S. 13; auch 2012b sowie 2014). Diese Position bekräftigt auch die im Juni 2014 in Wien abgehaltene tarif- und sozialpolitische Konferenz der Organisation: „Lohnerhöhungen, die über den Produktivitätszuwachs und Inflationsausgleich hinausgehen, sind wirtschaftlich sinnvoll und sozial verantwortlich, weil eine Umverteilung von Kapital-zu Lohneinkommen der beste Weg zur Konjunkturbelebung ist" (IndustrieALL 2014).

Eine strukturelle Aufwertung der tarifpolitischen $\mathrm{Zu}$ sammenarbeit zwischen den nationalen Mitgliedsgewerkschaften nimmt ihren Anfang im Jahr 1994 in einer hierzu eingerichteten Arbeitsgruppe nicht zuletzt unter Bedingungen der beschlossenen Wirtschafts- und Währungsunion. Die Zuordnung tarifpolitischer Koordination an die internationalen Sekretariate, so ein Vertreter von IndustriALL, bedingte für ihre operative Umsetzung jedoch häufig einen Mangel an Expertise: „Because traditionally, if you are talking about the last century, all international business was done by the international departments. But basically, and a bit harshly and perhaps not $100 \%$ correct, those people often did not really know the inside of the business." (Interview)

Die Übertragung der Koordinierungsaufgaben an den internationalen Tarifpolitischen Ausschuss Mitte der 1990er Jahre stellt damit einen Wendepunkt in der Geschichte der Lohnkoordinierung dar. Die Eucob@n-Datenbank bildet hierbei einen Bezugsrahmen, in den Metallgewerkschaften aus allen europäischen Ländern ihre Tarifergebnisse einspeisen sollen. In der Praxis verfolgt IndustriALL hierbei einen lediglich weichen Koordinierungsanspruch, dessen Verbindlichkeitsgrad sich aus einer ", moralischen Selbstverpflichtung" ableitet (Henning 2013, S. 163). Als mittel-oder langfristiges Ziel dieser Abstimmungsaktivitäten im Rahmen "transnationaler Kommunikationsräume" (ebd.) ist die Strategie explizit auf die ferne Zukunft hin orientiert: „Hinter diesem regionalen gewerkschaftlichen Vernetzungsansatz steht die (erfahrungsgesättigte) Überlegung, dass sich die Europäisierung der Tarifpolitik letztlich nur als Ergebnis eines Prozesses der zunehmenden gegenseitigen Kommunikation, Vertrauensbildung und Verständigung

5 Eine umfassendere theoretische Rahmung an der Schnittstelle von pragmatistischer Handlungstheorie (Ansell 2011) und Skandinavischem Institutionalismus (Brunsson 1989) findet sich bei Seeliger (2017). 
der nationalen Tarifakteure herstellen wird und dass es wichtig ist, die dazu erforderlichen strukturellen Voraussetzungen zu schaffen" (ebd., S. 274).

$\mathrm{Da}$ IndustriAlLL in der Lohnkoordinierung keinerlei Weisungsbefugnis besitzt, verbleibt die letztliche Entscheidung über Höhe und Inhalt tariflicher Forderungen in der Praxis bei den nationalen Mitgliedsorganisationen. Über die (Nicht-)Einhaltung der Koordinierungsregel (Lohnforderungen im Einklang mit Inflationsrate und Produktivitätszuwachs) wird anschließend im Tarifpolitischen Ausschuss reflektiert. Die Sanktionsmacht des Ausschusses beschränkt sich hierbei jedoch auf den moralischen Appell an die Mitgliedsorganisationen.

Zur praktischen Relevanz der Koordinierungsregel bekennen sich die befragten Regionalvertreter der IG Metall, die selbst Tarifverhandlungen führen, mit deutlichen Worten: „Also, ich bin immer ehrlich gesagt sehr stolz darauf gewesen und freue mich auch, dass IndustriALL letztendlich dann beschlossen hat, dass das keine Guideline ist, diese Koordinierung, sondern eine Regel ist." (Interview)

\subsection{Zur Lohnentwicklung der Metall- und Elektroindustrie von 1998 - 2013}

Kontrastieren wir die programmatischen Positionierungen der Funktionäre mit der tatsächlichen Lohnentwicklung, zeigt sich eine deutliche Diskrepanz. Die Einordnung dieses Befundes erfordert einen Blick auf die tarifpolitischen Rahmenbedingungen am deutschen Standort:

Die langfristige Entwicklung der Löhne weist seit den 1980er Jahren ein lediglich moderates Wachstum unterhalb der Schwelle von Inflation und (sektoraler wie gesamtgesellschaftlicher) Produktivität auf. Dieser Umstand bedingte nicht nur eine wesentliche Umverteilung zugunsten des Kapitals, sondern auch die Tatsache, dass in Deutschland als einzigem EU-Land 2014 ein niedrigerer Reallohn gezahlt wurde als zehn Jahre zuvor (Bispinck/Schulten 2014). Eine zunehmende Lohndifferenzierung zwischen Industrie- und Dienstleistungssegmenten bedingte weiterhin eine Abnahme der horizontale Koordinierung des deutschen Lohnfindungssystems. Einem leichten Aufschwung der folgenden Jahre zum Trotz benennt Bispinck (2011, S. 49) den Zeitraum zwischen 2001 und 2011 als ,tarifpolitisch verlorenes Jahrzehnt". Für diese Entwicklung lassen sich drei Ursachenkomplexe benennen:

(1) Der Einflussverlust intermediärer Organisationen macht sich für die Gewerkschaften in Form eines Absinkens ihrer Mitgliedschaft um 12\% (d. h. 900.000 Mitglieder) zwischen 2004 und 2014 bemerkbar. Das in Teilen der Politischen Ökonomie nach wie vor vorherrschende Bild eines stark koordinierten, korporatistischen Systems basierte demnach wesentlich auf einer Analyse der 1970er und 80er Jahre. Die Intermediarität der Gewerkschaften steht vor diesem Hintergrund infrage (Dörre 2016).
(2) Ein zweiter Trend zur Verbetrieblichung tariflicher Auseinandersetzungen nahm seinen Anfang zu Beginn der 1980er Jahre, als neue Arbeitszeit- und Beschäftigungsmodelle ,erste Bruchstellen im System der überbetrieblichen Lohnfindung" (Streeck 2013, S. 14) zu versinnbildlichen beginnen. Über die systematische Öffnung von Flächentarifverträgen zu Beginn der 1990er Jahre fühtt dieser Weg über die 1993 eröffneten „Härtefallklauseln" für ostdeutsche Betriebe schließlich zum Pforzheimer Abkommen des Jahres 2004. Diese Vereinbarung ermöglicht es Betriebsleitungen, mit der Zustimmung der Tarifparteien (IG Metall und Gesamtmetall) Negativabweichungen vom vereinbarten Lohn zu gestalten. Vor diesem Hintergrund haben sich Tarifabweichungen über das letzte Jahrzehnt in fast allen Branchen "zu einem festen Bestandteil des bundesdeutschen Tarifsystems entwickelt" (Haipeter 2011, S. 31).

(3) Ein dritter Ursachenkomplex findet sich schließlich in den Arbeitsmarktreformen und der neuen Rolle des Staates (Streeck 2009). Die Hinwendung der traditionellen zur "Markt-Sozialdemokratie" hat, so Nachtwey $(2009$, S. 72), „dem Kapital in doppelter Weise zugearbeitet". Zum einen dienten die arbeitsmarktpolitischen Reformen der Schröder-Regierung der Senkung der Lohnkosten. Verstärkt wurde dieser Trend, weil die Volizeitbeschäftigung zwischen 2001 und 2010 von 25,5 Mio. auf 23,6 Mio. zurückgegangen war, während die Zahl der meist niedriger bezahlten Teilzeitarbeitsplätze im selben Zeitraum von 10 auf 12,5 Mio. anstieg. Der Niedriglohnsektor umfasst hierbei kontinuierlich 22 bis $24 \%$ der in Deutschland legal Beschäftigten. Gleichzeitig war der Anteil flexibler, nichtstandardisierter Arbeitsverhältnisse an der Gesamtbeschäftigung 2013 auf 38\% (Ost), bzw. 39\% (West) gestiegen (Dörre 2016, S. 355). Eine Tendenz zur Auflösung des wohlfahrtsstaatlichen Klassenkompromisses lag zweitens im Rückbau der sozialen Sicherungssysteme, die unter der Bedingung zunehmender öffentlicher Verschuldung sowie einer sinkenden Staatsquote ihre dekommodifizierende Wirkung zu verlieren drohte. Der Erschöpfung staatlicher Gestaltungskapazität begegnete man wirtschaftspolitisch mit einem restriktiven Management des Arbeitsangebots (Streeck 2009, S. 94).

Wie in der Gesamtwirtschaft finden sich auch in der Metallund Elektroindustrie über die letzten Jahrzehnte hinweg verschiedene Ursachen einer moderaten Lohnentwicklung. Hierzu zählen u. a. die Abnahme der Tarifbindung, die Öffnung flächentariflicher Vereinbarungen, der Abbau übertariflicher Entgelte (z. B. im Tausch gegen Standortsicherung) sowie die Ausweitung atypischer Beschäftigung. Symptomatisch für diese Entwicklung diagnostiziert Haipeter (2014) einen dreifachen Trend:

- Eine Modernisierung bedingt erstens der zwischen 2002 und 2004 in Kraft getretene Entgeltrahmentarifvertrag mit Neuerungen in der Eingruppierung, der Einführung neuer Statuskomponenten wie Kooperation und Flexibilität, einer stärkeren Orientierung am Zielentgelt als Ent- 
TABELLE 1

Lohnentwicklung im Flächentarifvertrag der deutschen Metall- und Elektroindustrie im Zeitraum 1998-2013

Angaben in Prozent

\begin{tabular}{|c|c|c|c|c|c|c|c|c|c|c|c|c|c|c|c|c|c|}
\hline Jahr & 1998 & 1999 & 2000 & 2001 & 2002 & 2003 & 2004 & 2005 & 2006 & 2007 & 2008 & 2009 & 2010 & 2011 & 2012 & 2013 & $\mathrm{DA} * *$ \\
\hline Zielinflationsrate EZB & 2 & 2 & 2 & 2 & 2 & 2 & 2 & 2 & 2 & 2 & 2 & 2 & 2 & 2 & 2 & 2 & \\
\hline Inflationsrate Deutschland & 1 & 0.6 & 1.4 & 2 & 1,1 & 1,6 & 1,6 & 1,5 & 1,5 & 2,3 & 2,6 & 0,3 & 1,1 & 2,1 & 2 & 1,5 & \\
\hline Produktivitätszuwachs Metallsektor & 5,1 & $\cdot$ & 10.1 & 2 & 1,9 & 2,2 & 4 & 4,8 & 6,4 & 5,1 & $-0,3$ & $-11,9$ & 10,4 & 5,9 & $-1,9$ & -0.7 & \\
\hline $\begin{array}{l}\text { Zielmarke } 1 \text { berechnet aus Produktivitätszuwachs und } \\
\text { Zielinflationsrate der EZB }\end{array}$ & 7.1 & * & 12.1 & 4 & 3,9 & 4,2 & 6 & 6,8 & 8,4 & 7,1 & 2 & 2 & 12,4 & 7,9 & 2 & 2 & \\
\hline $\begin{array}{l}\text { Zielmarke } 2 \text { berechnet aus Produktivitätszuwachs und } \\
\text { nationaler Inflationsrate }\end{array}$ & 6,1 & $*$ & 11,5 & 4 & 3 & 3,8 & 5,6 & 6,3 & 7.9 & 7,4 & 2,6 & 0,3 & 11,5 & 8 & 2 & 1,5 & \\
\hline Forderung & & 6,5 & 5,5 & & 6,5 & & 4 & & 5 & 6,5 & & 8 & * & & 6,5 & & \\
\hline Abschluss & 2,5 & 3,2 & 3 & 2,1 & 3.1 & 2,6 & 1,5 & 2 & 3 & 4.1 & 2,1 & 2,1 & 0 & 2,7 & 4,3 & 3,4 & \\
\hline Abweichung von Zielmarke 1 & $-4,6$ & $*$ & -9.1 & -1.9 & $-0,8$ & $-1,6$ & $-4,5$ & $-4,8$ & $-5,4$ & -3 & 0,1 & 0,1 & $-12,4$ & $-5,2$ & 2,3 & 1.4 & $-3,3$ \\
\hline Abweichung von Abweichung von Zielmarke 2 & $-3,6$ & * & $-8,5$ & -1.9 & 0,1 & $-1,2$ & $-4,1$ & $-4,3$ & $-4,9$ & $-3,3$ & -0.5 & 1,8 & $-11,5$ & $-5,3$ & 2,3 & 1,9 & $-2,9$ \\
\hline
\end{tabular}

- Keine Angabe.

** Durchschnittliche Abweichung

Quelle: Forderungen IG Metall: http:/funww.gesamtmetall.de/gesamtmetall/meonline.nsffid/Page-Tarifchronik-Tarifentwicklung-seit-1990/\$file/Tarifentwicklung-1990-2012.pdf: Produktivität: http://www.gesamemetall. de/gesamemetall/meonline.nsfíd/PageJahreszahlen-in-langen-Zeitreihen_DE; Abschlüsse IG Metall:WSI-Tarifarchiv.

lohnungsstandard sowie einer Erhöhung des Leistungsentgelts im Verhältnis zum Grundentgelt.

- Zweitens stellt eine Variabilisierung fixierte Lohnkomponenten weiter zur Disposition, indem sie umsatz- und/ oder gewinnabhängige Standards stärkt.

- Und drittens tritt eine Fragmentierung in erster Linie in Form einer zunehmenden Lohnspreizung in Erscheinung.

Tabelle 1 illustriert die Lohnentwicklung in diesem Bereich über den Zeitraum von 1998 bis 2013 hinweg im Verhältnis zur Koordinierungsregel von IndustriALL Europe als aggregiertes Ergebnis aller flächentarifvertraglichen Lohnverhandlungen. Die freien Kästchen in der Zeile „Forderung“ beschreiben Zeiträume, in denen der abgeschlossene Tarifvertrag weiterhin gültig war. Dass auch im Jahr nach den Tarifverhandlungen ein Lohnanstieg zu verzeichnen ist, liegt an der Laufzeit der abgeschlossenen Verträge, die die Dauer eines Jahres überschreiten. Nicht berücksichtigt sind verschiedene praktische Abweichungen vom Flächentarifvertrag. Positivabweichungen bedingen hier variable Lohnelemente wie Einmalzahlungen und andere Boni. Negativabweichungen entstehen durch Öffnungsklauseln. Eine klare Bezifferung der realen Abweichung von der Regel ist ausgehend von der aktuellen Datenlage nicht möglich. Aufgrund der unterschiedlichen Modi, nach denen die Lohn forderungen ermittelt werden können, enthält die Tabelle Kalkulationen unter Bezug auf 1) die nationale Inflationsrate und 2) die Zielinflationsrate der $\mathrm{EZB}^{6}$

Wie die Darstellung zeigt, unterschreitet die reale Lohnentwicklung die Koordinierungsregel fast über den gesamten Zeitraum hinweg. Misst man die durchschnittliche Abweichung unter Bezug auf die nationale Inflationsrate (1,5\% für den untersuchten Zeitraum), beträgt die durchschnittliche Abweichung vom Regelwert minus 2,9\%. Im Falle einer Setzung der EZB-Rate fällt sie mit minus 3,3\% leicht stärker aus. Zuwächse oberhalb des Richtwertes lassen sich lediglich in den Jahren 2008, 2009, 2012 und 2013 beobachten. Zwar entsprechen die Eingangsforderungen (die Nullrunde des Jahres 2010 ausgenommen) allesamt zumindest dem in der Koordinierungsregel festgeschriebenen Wert. Wenn man diese hätte durchsetzen können, wäre eine Einhaltung der Regel wahrscheinlich gewesen. Da die Verhandlungsergebnisse erfahrungsgemäß aber wesentlich von den Ausgangsforderungen abweichen, deutet dies auf eine grundsätzliche Bereitschaft hin, die Koordinierungsregel in der Praxis aufzuweichen. Insgesamt ist das programmatisch stark betonte Ziel einer Erfüllung der Regel somit als verfehlt zu beschreiben. Während weichere Indikatoren wie die Intensivierung des Austausches oder die etwaige Berücksichtigung tarifvertraglicher Elemente wie etwa Weiterbildungsansprïche oder betriebliche Rentenregelungen durchaus als erfolgreiche Resultate anhaltender Koordinierungsinitiativen gesehen werden können, zeigt sich in der unmittelbar lohnpolitischen Dimension eine deutliche Diskrepanz zwischen Anspruch und Wirklichkeit.

6 Variabilisierung und die Fragmentierung verunkiaren die Abbildung der Lohnentwicklung in dem Sinne, dass sich der Zeitpunkt der Entlohnung verschieben kann. Weiterhin erhöht sich so auch die Bedeutung nicht unmittelbar monetärer Lohnelemente wie Weiterbildungsmaßnahmen oder Rentenansprüche. Schließlich sind auch Lohnverminderungen durch betriebliche Öffnungskjauseln nicht abgebildet. 


\subsection{Zur Praxis der Lohnkoordinierung}

Den Vorwurf absichtlicher Lohnzurückhaltung bezeichnet einer der befragten Regionalvertreter als "riesigen Schmarrn" und fügt hinzu: „Naja, es gibt sozusagen den Vorwurf in der Frage, dass die Tarifabschlüsse zu gering sind. Aber auch da ist die Frage, ist das tatsächlich anders möglich. Also, ich kenne die Debatte nicht, wo man sagt, okay, eigentlich könnten wir $5 \%$ haben, aber lass uns mal nur $3,8 \%$ abschließen." (Interview)

Als Ursache benennen die Repräsentanten der Organisation und an dieser Stelle einer der befragten Regionalvertreter die ungünstigen Rahmenbedingungen der letzten Jahre. Vor diesem Hintergrund gibt sich auch ein anderer Vertreter mit dem Arbeitsschwerpunkt Tarifpolitik mit Blick auf die Erfolgschancen der Lohnkoordinierung unter Bedingungen heterogener Lohnfindungssysteme im Euroraum nicht allzu optimistisch: „Das Problem ist hier einfach, also eins der Grundprobleme ist bei dieser Lohnkoordinierung: Wie soll das bitte funktionieren? Wir haben kein Rezept dafür und an der Stelle muss man sagen, wir haben es versucht in den letzten Jahren." (Interview)

In diesem Sinne relativiert einer der regionalen Tarifsekretäre die praktische Relevanz des europäischen Referenzrahmens für die realen Lohnverhandlungen: „Also, der $\mathrm{Be}$ gründungszusammenhang, den man eigentlich verinnerlicht hat, [ist] auch nach außen hin so darzustellen. Und dann hat es, wenn wir in der Bewegung sind, nicht mehr so eine hohe Bedeutung. Also, du kannst dich beim Warnstreik nicht da hinstellen und sagen, wir machen es deshalb, weil unsere europäischen Freunde das benötigen." (Interview)

Die gleiche Konstellation beschreibt auch sein Kollege aus einem anderen Tarifgebiet „Europa ist da nicht so präsent, wie sich das vielleicht in den Broschüren, oder wie man sich das in der Wirklichkeit so vorstellt. Wie gesagt, da sitzt keiner im Hinterzimmer und hat den europäischen Rechner auf, tippt das ein und sagt, geht nicht oder geht. Sondern das ist eher so ein Punkt, wo man sagt, wir müssen uns auch dazu rechtfertigen, wir müssen das einfließen lassen, aber in der praktischen Arbeit habe ich jetzt keinen Ordner in der Tarifrunde 2015, wo ich jetzt Europa stehen habe." (Interview)

Bezogen auf die praktische Abstimmung im Sinne einer gemeinsamen Regel schlussfolgert der Sprecher aus seinen Erfahrungen als Delegierter auf der gemeinsamen Tarifpolitischen Konferenz von IndustriALL im Juni 2014: „Können Sie sich vorstellen, dass dieser Club ein gemeinsames Verhandlungsgremium hat und sagt, okay, ich verhandele das jetzt und aufgrund der unterschiedlichen Systeme von Rumänien, Bulgarien, über die Slowakei, Österreich, Deutschland bis hoch nach Norwegen eine gemeinsame Linie zu finden? Was ist denn jetzt sozusagen die richtige Lohnsumme, was ist denn jetzt die richtige Arbeitszeit?" (Interview)

Weiterhin verneint der Interviewpartner auch die Teilnahme nationaler Vertreter an den Tarifverhandlungen im jeweils angrenzenden Ausland und verweist so auf die
Nicht-Praktizierung eines wesentlichen Bestandteils der Koordinierungsinitiative. Zusätzliche Probleme für die Koordinierungspraxis offenbaren sich aber auch bei der Analyse der Erhebung tarifpolitischer Ergebnisse in der Eucob@n-Datenbank. So besteht erstens keinerlei Übereinstimmung in der Frage, welche Inflationsrate zugrunde zu legen ist - die der EZB oder die jeweils nationale. ${ }^{7}$ Wie die IG Metall (2011) bereits in ihrem Bericht zur Wirkung der Koordinationsregel ermittelt, existiert zweitens bis heute keine offizielle europäische Statistik mit harmonisierten Tarifdaten. Hierfür gibt es unterschiedliche Gründe: Zum einen benennt IndustriaLL (2014, S. 10) „Probleme bei der Datenerhebung ergeben sich hinsichtlich der Zuverlässigkeit der Meldungen." Dass nationale Mitgliedsorganisationen keine ausreichenden Informationen über die dortigen Tarifentwicklungen liefern, verhindert eine vergleichbare Darstellung im europäischen Maßstab. Doch auch wenn diese Daten lückenlos vorlägen, wäre eine entsprechende Aufbereitung schwierig. Denn die Organisationsgebiete der jeweiligen Gewerkschaften unterscheiden sich voneinander: Während etwa die IG Metall einen eigenen Tarifabschluss für die Metall- und Elektroindustrie erwirkt, spielt sich die Lohnfindung in anderen Ländern über andere Segmente hinweg ab.

Vor diesem Hintergrund verdeutlicht sich eine Diskrepanz in der politischen Orientierung der IG Metall: Die im Rahmen der tarifpolitischen Selbstdarstellung der IG Metall formulierten Leitsätze zur Lohnkoordinierung spielen in der praktischen Auseinandersetzung nicht die Rolle, die ihnen rhetorisch zugedacht wird. Diese Einschätzung wird von einem internationalen Vertreter auf den Punkt gebracht: "Das ist alles wunderbar nett. Ist ein Riesenaufwand, Eucob@n, was die da leisten, was die an Daten sammeln. Aber ich kann sagen, ich kenne Brüssel jetzt über 30 Jahre. Ich habe den Eindruck, okay, man tut da einiges. Aber wenn es um nationale Interessen geht, interessiert das alles nicht. $\mathrm{Da}$ bin ich also absolut skeptisch, ich halte das mittlerweile für eine Riesenheuchelei." (Interview)

Die Orientierung an der Lohnformel stellt für ihn lediglich eine "offizielle Sprachregelung" dar. Tatsächlich sei es aber so "dass Leute letztlich ihre nationale Hose sehen. Oder ihr Hemd sehen, und das ist ihnen näher als alles andere." (Interview)

Bis hierhin scheint es also so, als ließe sich die Initiative zur Lohnkoordinierung als Strategie und mit den Worten des IndustriALLL-Vertreters als systematisch in Kauf genommener Widerspruch oder - wie es der Interviewpartner formuliert - sogar Heuchelei erklären. Aber stellt dies wirklich eine ausreichende Erklärung dar? Hinter der anhalten-

7 Und auch über die Ermittlung Letzterer besteht keineswegs Einigkeit. Während z. B. in den Niederlanden die Herstellerpreise hinzugezogen werden, verlässt man sich in Belgien auf den sog. "Gesundheitsindex" und in Deutschland auf den „Deflationierer" der Verbraucherpreise 
den Verfolgung einer (nominellen) Koordinierungsstrategie erkennt einer der regionalen Tarifsekretäre den Beweggrund, „dass man nach außen programmatisch deutlich macht, was wir da wollen. Und dass im Alltag das eine oder andere dann hinten runterfältt, das ist dann keine böse Absicht oder Vernachlässigung oder irgendwas." (Interview)

Lohnkoordinierung dient aus dieser Sicht also nicht der tatsächlichen Abstimmung von Löhnen (oder entsprechender Forderungen), sondern als programmatische Aufforderung dazu. Dementsprechend beschreibt auch der andere Tarifsekretär die Diskrepanz ,zwischen Theorie und Praxis " der Initiative. Zwar sei die praktische Wirklichkeit „oft komplizierter als das, was am grünen Tisch gedacht wird." (Interview). Gleichzeitig benötige die tarifpolitische Praxis aber auch eine auf die Zukunft gerichtete Programmatik, welche sich aus der Theorie ergebe: "Wenn ich alles nur auf Praxis nehme, und sage, die gemeinsame große Idee ist mir egal, das wird auch nicht funktionieren. Aber dieses Spannungsfeld, das macht ja eigentlich das aus meiner Sicht erst so interessant, sozusagen, aus gewissen Gründen, verwehre ich jetzt der Praxis auch etwas. Oder ich mache es dann eben nicht. Oder ich versuche, es nochmal besser zu machen, als ich es heute hinkriege. Aber ich kann mich nicht nur auf der einen Seite bewegen. Ich muss sozusagen das ganze Feld auch mit betrachten." (Interview)

Diesen programmatischen Charakter der Zusammenarbeit stellt auch ein ehemaliger Mitarbeiter des EMB heraus: „Das ist mit den Parteiprogrammen ja auch nicht anders. Da steht ja auch meist Gutes drin. Ich finde, so ein Programm zu entwickeln, das ist auch insofern Klasse, dass wir überhaupt mal diskutieren." (Interview) In diesem Sinne betrachtet ein Interviewpartner die Initiative zur Lohnkoordinierung auch "nicht als MühIstein, der mir immer um den Hals gelegt wird, sondern [...] eher als gemeinsame, sinnstiftende Idee, zu sagen, es ist schon richtig." (Interview)

Dass die Koordinierung von Lohnabschlüssen allerdings für immer auf den Status einer reinen Idee zu reduzieren sei, ist damit, so der Tenor der Interviews, nicht gesagt: Auf die Frage, ob eine vor allem symbolische Koordinierung ihm angesichts der unmittelbaren Problemsituation nicht als frustrierendes Unterfangen erscheine, erläutert ein IndustriALL-Vertreter seine programmatische Orientierung als langfristig angelegte Politikauffassung, welche in erster Linie auf Hartnäckigkeit und Kontinuität bei der Verfolgung der angestrebten Ziele beruhe: „You want early retirement at 58 in your sector. And you ask it from the employers. And they don't give it to you. In two years' time, we come back and we want early retirement with 58 . And if we don't succeed, we come back and want early retirement with 58 . Until finally, we succeed. It is the objective in collective bargaining." (Interview)

Wahrend verbindliche gesetzliche Regelungen "at the moment" (sic!) nicht möglich seien, könnten diese über einen längeren Zeitraum hinweg durchaus angestrebt wer- den. Diesen Ansatz beschreibt er als "working towards the future and guiding it." (Interview) Aktuelle Initiativen zur Lohnkoordinierung dienten vor diesem Hintergrund der perspektivischen Etablierung einer europäischen Verhandlungsarena - und eben anders als gesagt nicht der Koordinierung von Löhnen in der Gegenwart. Die Möglichkeit einer Koordinierung im Sinne der Regel kann für die $\mathrm{Zu}$ kunft allerdings keineswegs ausgeschlossen werden. Und genau hieraus beziehen die Initiativen einen wichtigen Teil ihrer Legitimation.

\subsection{Zusammenfassung der empirischen Befunde}

Während die Koordinierung nationaler Lohnpolitiken im europäischen Metallsektor seit Mitte der 1990er Jahre angestrebt wird und eine Beeinflussung des lohnpolitischen Diskurses im Sinne eines lohngetriebenen Wachstumsmodells vor dem Hintergrund der European Economic Governance (Pernicka 2015) festzustellen ist, ist der Erfolg dieser Initiative gemessen an ihrem nominellen Ziel (gleichmäßige Lohnentwicklung mindestens in Höhe von Inflationsrate und Produktivitätszuwachs) gering.

Die Abstimmung der Lohnentwicklung im Einklang mit der Koordinierungsregel konnte, dies zeigt sich in Tabelle I, über den Untersuchungszeitraum hinweg nicht erreicht werden. Als Ursachen für das Scheitern der Initiative lassen sich verschiedene institutionelle Kontextfaktoren erkennen die Heterogenität der Lohnfindungssysteme in der Eurozone sowie die begrenzte tarifpolitische Durchsetzungsfahigkeit der IG Metall unter Bedingungen von Mitglieder verlust, abnehmender Tarifbindung und Arbeitsmarktreformen. ${ }^{8}$ Dass IG Metall und IndustriALL auch unter diesen widrigen Rahmenbedingungen an dem Projekt festhalten, erscheint widersprüchlich mit Blick auf eine Reihe operativer Defizite der Initiativen:

(1) Aufgrund der unterschiedlichen tarifpolitischen Zuständigkeiten der zahlreichen nationalen Gewerkschaftsorganisationen gewährleisten die Daten keine Vergleichbarkeit der Tarifentwicklung.

(2) Es herrscht keine allgemeine Übereinkunft darüber, welche Inflationsrate zur Umsetzung der Regel hinzugezogen werden soll - die jeweils nationale oder die von der EZB angestrebte. Dieselbe Unklarheit besteht im Hinblick auf die Wahl der Produktivitätsentwicklung. Unter den europäischen Metallgewerkschaften herrscht über den Untersuchungszeitraum hinweg keinerlei Einigkeit darüber, ob diese in Bezug auf den jeweiligen Wirtschaftssektor oder die nationale Gesamtwirtschaft zu ermitteln ist.

8 Inwiefern eine Strategie der Lohnzurückhaltung die ausschlagebene Ursache ist (vgl. Höpner 2013, S. 6), kann hier nicht beantwortet werden. 
(3) Die im Rahmen der Koordinierungsinitiative vorgesehene Entsendung nationaler Vertreter zur Teilnahme an den Tarifverhandiungen von Schwestergewerkschaften aus den Nachbarländern existiert zwar als Idee, wird aber nicht (oder zumindest nicht flächendeckend) praktiziert.

Vor diesem Hintergrund ist die Haltung von IG Metall und IndustriALI zur Lohnkoordinierung als ambivalent einzuschätzen. Zwar fällt das Bekenntnis zu ihrer Notwendigkeit im Rahmen verschiedener Publikationen sowie in Äußerungen der interviewten Vertreter sehr eindeutig aus. In der Praxis aber erscheint, auch dies zeigt sich an den zitierten Passagen, die Bedeutung der Koordinierungsregel als eher untergeordnet. Eine Forderung in Höhe der Lohnkoordinjerungsregel lässt sich zwar in fast allen untersuchten Tarifrunden finden. Da aber wohl kaum eine Gewerkschaft (und historisch betrachtet auch nicht die IG Metall) ihre Eingangsforderungen 15 Jahre in Folge durchzusetzen vermag, deutet die zu erwartende Diskrepanz zwischen Forderung und Abschluss auf die Schlussfolgerung hin, dass das Scheitern der nominellen Forderungen, die die Regeln der Lohnkoordinierung durchaus reflektiert haben, letztlich einkalkuliert wird. Gegenüber dem eigenen formulierten Anspruch müssen mit Blick auf die Realität der Koordinierungspraxis also wesentliche Abstriche gemacht werden. Als Ergebnis lässt sich festhalten, dass es sich bei den koordinierten Werten keinesfalls um tatsächliche Lohnabschlüsse, sondern lediglich die jeweils geltend gemachten Forderungen handelt. Zugespitzt ausgedrückt könnte man insofern an Stelle von Lohnkoordinierung konsequenterweise auch eher von Lohnforderungskoordinierung sprechen. Wie aber ist diese Diskrepanz abschließend zu bewerten?

\section{Fazit}

Die empirische Analyse zeigt - zumindest oberflächlich betrachtet - dass entgegen aller programmatischen Ziele die Umsetzung EU-weiter gemeinsamer tarifpolitischer Positionen in der Vergangenheit gescheitert ist. Dieser Befund scheint euroskeptischen Positionen, wie sie schon seit geraumer Zeit zu vernehmen sind (Höpner/Schäfer 2010; Scharpf 2009), Recht zu geben. Zu groß sind aus dem Blickwinkel der Vergleichenden Politischen Ökonomie die Unterschiede zwischen den Ländern, und die auf Veränderung und Angleichung hinwirkenden Potenziale der beteiligten Akteure erscheinen als zu gering. Allerdings offenbart der hier verfolgte handlungstheoretische Ansat $/$ eine andere Perspektive - und erzählt damit eine andere Geschichte: Denn gegen eine "strukturalistische oder deterministische Sicht", so bemerkt mit Kowalksy (2010, S. 139) ein Vertreter der eurooptimistischen Seite, gelte es, "festzuhalten, dass europäische Initiativen, die Konflikte hervorrufen, deutliches Politisierungsund damit Veränderungspotenzial haben."
Um den Widerspruch im Verhalten von IG Metall und IndustriALL verstehen zu können, gilt es, die konkurrierenden Handlungslogiken in Betracht zu ziehen, die die politische Orientierung von Gewerkschaften bestimmen: Während Schmitter und Streeck (1999) mit der Mitgliederlogik die unmittelbaren Interessen der Mitglieder an Lohn. zuwächsen und Arbeitsplatzsicherheit herausstellen, unterstreichen Frege und Kelly (2004) einen Status von Gewerkschaften als "wertrationale Organisationen". Ein schlichtes Insistieren auf der Diskrepanz zwischen internationalistischem Reden und standortnationalistischem Handeln greift zum Verständnis des komplexen Mobilisierungsprozesses zu kurz.

An Stelle einer rein kalkulierten Kompensationsstrategie gelingt es den Organisationen auf diese Weise, ihre Handlungsfähigkeit auch unter widrigen Bedingungen zu erhalten. Wie gezeigt wurde, bedingt die multiple Einbindung der IG Metall in das Mehrebenensystem der EU verschiedene inkonsistente Erwartungen. Dass eine reale Erfüllung häufig als nicht umsetzbar erscheint, bedeutet jedoch nicht, dass ihre Vertreter keine derartigen Ambitionen verfolgen. In der dezidierten Zukunftsorientierung der Initiativen findet sich ein Moment, das über die Prämissen einer bloßen Entkoppelung hinausweist. Denn um zu einem späteren Zeitpunkt in der Lage zu sein, Lohnabschlüsse miteinander koordinieren zu können, so der Tenor unter den Befragten, müssen entsprechende Initiativen weiter aufrechterhalten werden, auch wenn sie gegenwärtig nicht zu den eigentlich gewünschten Ergebnissen führen. Was von außen betrachtet wie ein systematischer Misserfolg oder sogar als (Selbst-) Betrug wirken mag, erscheint unter Berücksichtigung der Akteursperspektive als (provisorisches) Zwischenergebnis unterschiedlicher miteinander verbundener Handlungsstränge. Dass jemand etwas tun will und soll, heißt nicht, dass er es auch kann - aber eben genauso wenig, dass er es nie können wird. Das Festhalten an der Lohnkoordinierung mit dem Versprechen einer Verbesserung der Initiative in der Zukunft erscheint vor diesem Hintergrund nicht nur als Kompensation gegenwärtigen Scheiterns, sondern auch als kreativer Umgang mit Unsicherheit

Aus einer eurooptimistischen Sicht kommt solchen symbolischen Bildern über die Zukunft der EU als politischem

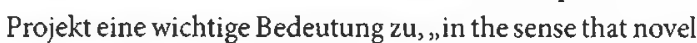
institutions do not emerge ex nihilo" (Sabel/Zeitlin 2012, S. 423): Die Etablierung relativ weitreichend gültiger und dauerhaft beständiger Regeln als Rahmenwerk eines politischen Systems erfordert (zumindest im Falle ihrer absichtsvollen Gestaltung) eine gemeinsame Vision unter den aktiv beteiligten Akteuren.

Wie Streeck (1998, S. 391) bemerkt, ist eine solche genuin optimistische Zukunftsorientierung grundsätzlich im Integrationsprojekt gewissermaßen strukturell angelegt: Damit die Mitgliedstaaten eine politische Gemeinschaft bilden können, müssen sie davon ausgehen, dass „diese sich zu einem ,Europa der Bürger' [...] entwickelt, oder ihr Integrationsprojekt wird aus Mangel an Unterstützung fehl- 
schlagen." Indem sie die Zielerreichung in die Zukunft verschieben, erhalten die Gewerkschaften ihre politische Handlungsfähigkeit. Über die reale Umsetzbarkeit der Lohnkoordinierung als tarifpolitischem Projekt ist damit freilich allerdings noch nichts gesagt. Diese Ambivalenz beschreibt auch Schulten (2005, S. 15): „ES ist gerade der Widerspruch zwischen der Hoffnung auf ein integriertes Europa als Gegengewicht zur neoliberalen Globalisierung einerseits und der praktischen EU-Politik, die vielfach selbst das neoliberale Projekt vorantreibt, andererseits, die das Verhältnis zwischen Gewerkschaften und europäischer Integration vielfach ambivalent erscheinen lässt."

Inwiefern die gegenwärtig mehr rhetorische als tatsächliche Koordinierung von Lohnforderungen ihr tarifpolitisches Potenzial für die Erreichung des Fernziels eines "Sozialen Europa" noch entfalten kann und eben nicht der Rationalisierung eines Integrationsprojektes dient, welches den meisten Gewerkschaften mehr schadet als nützt, wird die Zukunft zeigen.

\section{LITERATUR}

Ansell, C. (2011): Pragmatist democracy. Evolutionary learning as public philosophy, Oxford

Bispinck, R. (2011): Tarifpolitischer Jahresbericht 2010: Beschäftigungssicherung und gedämpfte Lohnentwicklung, http://www.boeckler.de/pdf/p_ta_jb_2010.pdf Bispinck, R./Dribbusch, H. (2011): Collective Bargaining. Decentralisation and crisis management in the German metalworking industries since 1990.

WSI-Discussionpaper (177), Düsseldorf

Bispinck, R./Schulten, T. (2006): Perspektiven einer gesamtwirtschaftlich orientierten Lohnpolitik, in: Schäfer, C./Seifert, H. (Hrsg.): Kein bisschen leise 60 Jahre WSI, Hamburg S. 253-270

Bispinck, R./Schulten, T. \{2014): Wages, collective bargaining and economic development in Germany, WSI-Diskussionspapier (191), http://www.boeckler.de/ wsi_5351,htm?produkt=HBS-005949\&chunk=1\&jahr=

Brunsson, N. (1989): The organization of hypocrisy: Talk, decisions and actions in organizations, Hoboken

Dörre, K. (2016): Die neue Konfliktformation. Klassen-Kämpfe in fragmentierten Arbeitsbeziehungen, in: Industrielle Beziehungen 23 (3), S. 348-365

EMB (Europäischer Metallgewerkschaftsbund) (2011): Work programme

2011-2015 of the European Metalworkers Federation, Brussels

Emirbayer, M./Mische, A. (1998): What is agency?, in: American Journal of Sociology 103 (4), S. $962-1023$

Eme, R. (2008): European unions. Labors quest for a transnational democracy, Itaca/London

Eme, R. (2013): Coordinating collective wage bargaining: A way of transnationa solidarity in Europe?, http://andreasbieler.blogspot.de/2013/10/coordinatingcollective-wage-bargaining.htmi

European Metalworkers' Federation (2011): Work programme 2011-2015 of the European Metalworkers Federation, Brussels

Frege, C./Kelly, J. (Hrsg.) (2004): Varieties of unionism, Oxford

Glassner, V. (2009): Two paths towards Europeanisation. The institutional and economic determinants of the transnationalization of collective bargaining on the European sector level, Dissertation Universität Wien

Gollbach, J. (2005): Europäisierung der Gewerkschaften. Praktische Ansätze im Spannungsfeld transnationaler Traditionen, Hamburg

Haipeter, T. (2011): Tarifabweichungen, Betriebsräte und Gewerkschaften -

Modernisierungschancen in lokalen Konflikten, in: Haipeter, T./Dörre, K. (Hrsg.) Gewerkschaftliche Modernisierung. Wiesbaden, S. 31-60

Haipeter, T. (2014): Das ungewisse Extra. Die Widersprüche erfolgsabhängiger Entgelte am Beispiel der Metall- und Elektroindustrie, Berlin

Hancké, B. (2013): Unions, central banks and EMU, Oxford

Henning, K. (2013): Europäische Integration und Gewerkschaften, Wiesbaden Höpner, M. (2014): Transnationale Lohnkoordination in einem System fester

Wechselkurse, in: WSI-Mitteilungen 67 (4), S. 317-319, https://www.boeckler.del wsi-mitteilungen_47210_47215.htm

Höpner, M./Schăfer, A. (2010): A new phase of European integration: Organized capitalisms in post-Ricardian Europe, in: West European Politics 33 (2), S. 344-368
Hom, G. A. (2010): Konstruktionsfehler des Euroraums, in: Bispinck, R. (Hrsg.): Zwischen "Beschäftigungswunder" und "Lohndumping"?, Hamburg, S. 59-79 IG Metall (2005): Europäische Koordinierung nationalerTarifpolitik, Frankfurt a. M. IG Metall (2011): Wirkung der Koordinierungsregel des EMB. Im Betrachtungszeitraum 2000 bis 2009, Frankfurt a. M.

IndustriALL (2012a): Politische Entschließung. Ind. 2/2012. Vom Gründungskongress der industriAll European Trade Union am 16.05. in Brüssel verabschiedet, http://industriall-europe.eu/committees/IP/PolPaper/industriAll-Political Resolution-DE.pdf

IndustriALL (2012b): IndustriALL European Trade Union Wage Coordination Rule, 27./28.11., Luxembourg

IndustriALL (2014): „Unsere Zukunft verhandeIn!” Gewerkschaftliche Strategien in Zeiten der Wirtschaftskrise. Dokument zurTarif-und sozialpolitischen Konferenz von IndustriALL, 12.-13. Juni, Wien

Joas, H. (1996): Die Kreativität des Handelns, Frankfurt a. M

Joas, H. /Beckert, J. (2001): Action theory, ins:Turner, J. H. (Hrsg.): Handbook of sociological theory, Wiesbaden, S. 269-285

Johnston, A. (2009): Wage policy in Austria and the Netherlands under EMU. A change in performance or the continuation of the status-quo?, ETUI Working Paper 7/2009, http://Www.etui.org/Publications2/Working-Papers/Wage-policyin-Austria-and-the-Netherlands-under-EMU

Kowalsky, W. (2010): Gewerkschaften und Europa, in: IPG 3/2010, S. $128-144$ Kühl, St. (2011): Organisation. Eine sehr kurze Einführung, Wiesbaden Marginson, P./Sisson, K. (2004): European integration and industrial relations, New York

Marginson, P./Traxler, F. (2005): After enlargement: Preconditions and prospects for bargaining coordination, in: Transfer 11 (3), S. 423-438

Meyer, J.W./Rowan, B. (1977): Institutionalized organizations: Formal structure as myth and ceremony, in: American Journal of Sociology 83 (2), S. 340-363 Müller, T. (2016): Tarifpolitische Koordinierung des Europäischen Gewerkschaftsbundes in Zeiten der Krise, in: WSI-Mitteilungen 69 (4), S. 309-312, https://www.boeckler.de/wsi-mitteilungen_65669_65677.htm

Müller, T./Schulten,T./van Gyes, G. (Hrsg.): Lohnpolitik unter europäischer "Economic Governance" Alternative Strategien für inklusives Wachstum, Hamburg

Müller-Jentsch, W. (2011): Gewerkschaften und Soziale Marktwirtschaft seit 1945, Stuttgart

Nachtwey, O. (2009): Marktsozialdemokratie. Die Transformation von SPD und Labour Party, Wiesbaden

Neusser, Ch. (2013): Pluralisierte Partnerschaften, Berlin

Pen, J (1964): Europäische Lohnpolitik, in: Gewerkschaftliche Monatshefte 14 (1), S. 581-585

Pernicka, S. (2015): Oynamiken von Macht und Gegenmacht in der europäischen Lohnkoordinierung, in: WSI-Mitteilungen 68 (8), S. 604-612, https://www.boeckler.de/wsi-mitteilungen_62503_62550.htm Rüb, St. (2009): Die Transnationalisierung der Gewerkschaften, Berlin

Sabel, C. F./Zeitlin, J. (2012): Experimentalism in the EU: Common ground and persistent differences, in: Regulation \& Governance 6 (3), 410-426

Scharpf. F. W. (2009): The double asymmetry of European integration - Or why the EU cannot be a Social Market Economy, MPIfG Working Paper 09/12, Berlin Schmitter, P. C./Streeck, W. (1999): The organization of business interests: Studying the associative action of business in advanced industrial societies, MPIFG Discussion Paper 99/1, Berlin

Schulten, T. (2004): Solidarische Lohnpolitik in Europa, Hamburg

Schulten, T. (2005): Gewerkschaften und europäische Integration. Aktuelle

Facetten eines ambivalenten Verhältnisses, in: Beerhorst, J./ Urban, H.-J. (Hrsg.): Handlungsfeld europäische Integration. Gewerkschaftspolitik in und für Europa, Hamburg, S. 14-36

Schulten,T. (2014): Europäische Lohnkoordinierung: Solidarisch oder wettbewerbsorientiert, in: WSI-Mitteilungen 67 (4), S. 314-316, https://www.boeckler.de/ wsi-mitteilungen_47210_47216.htm

Schulten, T./Bispinck, R. (Hrsg.) (1999): Tarifpolitik unter dem Euro. Perspektiven einer europäischen Koordinierung, Hamburg

Schulten, T./Müller, T. (2013): Ein neuer europäischer Interventionismus? Die Auswirkungen des neuen Systems der europäischen Economic Governance auf Löhne und Tarifpolitik, in: Wirtschaft und Gesellschaft 39 (3). 291 - 320 Schulten, T./van Gyes, G./Müller, T. (2016): Fazit: Europaweite Stärkung der Tarifvertragssysteme als Voraussetzung für ein inklusives Wachstumsmodell in Europa, in: Müller, T./Schulten, T./van Gyes, G. (Hrsg.): Lohnpolitik unter europäischer "Economic Governance". Alternative Strategien für inklusives Wachstum, Hamburg, S. 309-319 
Seeliger, M. (2017): Die soziale Konstruktion organisierter Interessen. Frankfurt a.M./New York

Sterkel, G./Wiedemuth, J. (2011): Neue Solidarische Tarifpolitik, in: Sozialismus $38(9)$, S. 26-32

Streeck, W. (1998): The Internationalization of Industrial Relations in Europe Prospects and Problems, in: Politics and Society 26 (4), S. $429-459$

Streeck, W. (1999): Korporatismus in Deutschland, Frankfurt a. M./New York

Streeck, W. (2009): Re-Forming Capitalism. Institutional Change in the German Political Economy, Oxford

Streeck, W. (2013): Gekaufte Zeit. Die vertagte Krise des demokratischen Kapitalismus, Berlin

Thelen, K. (2014): Varieties of liberalization and the new politics of social solidarity, Cambridge

Traxler, F./Brandl, B. (2009): Towards Europeanization of wage policy. Germany and the Nordic Countries, in: European Union Politics 10 (2), S. 177-201

Traxler, F./Brandl, B./Glassner, V./Ludvig, A. (2008): Can cross-border bargaining coordination work?, in: European Journal of Industrial Relations 14 (2).

S. $217-237$
AUTOR

MARTIN SEELIGER, Dr. ist wissenschaftlicher Mitarbeiter an der EuropaUniversität Flensburg. Arbeitsschwerpunkte: Gewerkschaftspolitik,

Organisationssoziologie, Soziale Ungleichheit.

(9) martin.seeliger@gmx.net 